\title{
MicroRNA-761 promotes the sensitivity of colorectal cancer cells to 5-Fluorouracil through targeting FOXM1
}

\author{
Shuguang Cao ${ }^{1}$, Limiao Lin ${ }^{1}$, Xuanping $\mathrm{Xia}^{1}$ and Hao $\mathrm{Wu}^{1}$ \\ ${ }^{1}$ Department of Gastroenterology, The Second Affiliated Hospital and Yuying Children's Hospital of Wenzhou Medical \\ University, Wenzhou 325000, Zhejiang, China
}

Correspondence to: Hao Wu, email: haowuse@126.com

Keywords: colorectal cancer, miR-761, 5-Fluorouracil, FOXM1

Received: June 06, 2017 Accepted: July 25, $2017 \quad$ Published: August 10, 2017

Copyright: Cao et al. This is an open-access article distributed under the terms of the Creative Commons Attribution License 3.0 (CC BY 3.0 ), which permits unrestricted use, distribution, and reproduction in any medium, provided the original author and source are credited.

\section{ABSTRACT}

Resistance to chemotherapy is a big challenge for treatment of patients with colorectal cancer; however; the mechanism underlying chemoresistance in colorectal cancer cell has not been elucidated. MicroRNAs (miRNAs) are new players in the development of drug chemoresistance. In our study, we indicated that overexpression of miR-761 promoted the sensitivity of colorectal cancer cells to 5-Fluorouracil (5-FU). miR-761 expression was downregulated in colorectal cancer cell lines and tissues. miR-761 expression was lower in patients with low grade than in patients with high grade. In additon, we showed that elevated expression of miR-761 suppressed colorectal cancer cell proliferation, cell cycle, colony formation and cell invasion. We identified that FOXM1 was a direct target gene of miR-761 in colorectal cancer cell. FOXM1 expression was upregulated in colorectal cancer tissues compare to the adjacent non-tumor tissues. MiR-761 expression was negatively associated with the expression of FOXM1 in colorectal cancer tissues. Elevated expression of FOXM1 suppressed the sensitivity of miR-761-overexpressing HT29 cells to 5-FU. We also indicated that FOXM1 overexpression promoted cell proliferation, cycle and invasion of miR-761-overexpressing HT29 cells. These data suggested that miR-761 played a tumor suppressor miRNA in colorectal cancer progression and reduced miR-761 expression might be a major mechanism for 5-FU resistance in colorectal cancer cell.

\section{INTRODUCTION}

Colorectal cancer (CRC) accounts for about 13\% of all tumors and the 2th leading cause of tumor-related death in developed countries [1-5]. Despite therapeutic strategies such as chemotherapy, surgery and radiotherapy that have been applied in CRC treatment, the prognosis of the colorectal cancer patients has not been satisfied over the last 20 years [6-9]. The molecular mechanism and pathological process of the colorectal cancer were complicated and was still not fully understood [10-12]. Therefore, it is important to elucidate the molecular mechanisms of colorectal cancer to find the new therapeutic strategies.

MicroRNAs (miRNAs) are small, evolutionarily conserved and short non-coding RNAs that work as posttranscriptional gene governor through targeting to the partially complementary sequences in $3^{\prime} \mathrm{UTR}$ of target mRNAs [13-17]. Numbers of studies have demonstrated that miRNAs play pivotal roles in diverse biological processes such as cell proliferation, differentiation, metastasis, angiogenesis, and invasion [18-22]. Increasing evidence demonstrated that miRNAs were dysregulated in many tumors including osteosarcoma, gastric cancer, hepatocellular carcinoma, lung cancer, gallbladder carcinoma and bladder cancer [23-28]. Recently, a lot of studies have demonstrated that miRNAs are a new player in the development of drug chemoresistance [29-31]. MiRNAs are deregulated expression in the chemoresistant and chemosensitive cells $[32,33]$.

Previous studies suggested that miR-761 acted important roles in the development and progression of various tumors [34-37]. However, the role of miR-761 in the colorectal cancer is still uncovered. In our study, we demonstrated that overexpression of miR-761 promoted the sensitivity of colorectal cancer cells to 5-FU. We demonstrated that the expression level of miR-761 was downregulated in colorectal cancer tissues and cell lines. 
We also indicated that elevated expression of miR-761 suppressed colorectal cancer cell proliferation, cell cycle, colony formation and cell invasion.

\section{RESULTS}

\section{miR-761 promoted the sensitivity of colorectal cancer cells to 5-FU}

The expression level of miR-761 was downregulated in colorectal cancer cells (HT29, SW480, SW620 and DLD-1) compared to normal colon epithelium cell line (FHC) (Figure 1A). qRT-PCR analysis indicated that miR761 mimic prommoted miR-761 expression both in the HT29 and SW480 cell (Figure 1B and 1C). The response of HT29 and SW480 cells to 5-FU was increased after treated with miR-761 mimic compared to the scrambletransfected cells (Figure 1D and 1E).

\section{miR-761 expression was downregulated in colorectal cancer tissues}

We then measured the miR-761 expression in the colorectal cancer tissues. Our data showed that miR-761 expression was downregulated in 29 colorectal cancer patients compare to the adjacent non-tumor tissues (Figure 2A). The expression of miR-761 was lower in colorectal cancer samples compare to the non-tumor samples (Figure 2B). In addition, miR-761 expression was lower in patients with low grade than in patients with high grade (Figure 2C).

\section{Elevated expression of miR-761 suppressed colorectal cancer cell proliferation}

We demonstrated that overexpression of miR-761 decreased cell proliferation in colorectal cancer cell lines HT29 and SW480 (Figure 3A and 3B). In addition, ectopic expression of miR-761 inhibited cyclin D1 expression in both HT29 and SW480 cell (Figure 3C and 3D). Moreover, elevated expression of miR-761 decreased HT29 and SW480 cell cycle (Figure 3E and 3F).

\section{Overexpression of miR-761 decreased colorectal cancer cell colony formation and invasion}

Ectopic expression of miR-761 suppressed HT29 and SW480 cell colony formation (Figure 4A and 4B). Moreover, we performed the invasion assay to measure cell invasion ability. Our data indicated that miR-761 overexpression decreased the HT29 and SW480 cell invasion (Figure 4C and 4D).

\section{FOXM1 was a direct target gene of miR-761}

To study the molecular mechanism of miR-761 in colorectal cancer cell, we used the website TargetScan database to identify potential target gene of miR-761.
The putative binding site of miR-761 and FOXM1 was shownAs shown in Figure 5A. Moreover, elevated expression of miR-761 suppressed luciferase activity of wild-type 3'UTR of the FOXM1 construct, but not in the mutated-type 3'UTR of the FOXM1 vector in HT29 and SW480 cells (Figure 5B and 5C). Furthermore, elevated expression of miR-761 inhibited the protein expression of FOXM1 in HT29 and SW480 cells (Figure 5D and 5E).

\section{FOXM1 expression was upregulated in colorectal cancer tissues}

We next detected FOXM1 expression in colorectal cancer tissues. Our data showed that FOXM1 expression was upregulated in 28 colorectal cancer patients compared to the adjacent non-tumor tissues (Figure 6A). The expression of FOXM1 was higher in colorectal cancer samples compare to the non-tumor samples (Figure 6B). In addition, miR-761 expression was negatively associated with the expression of FOXM1 in colorectal cancer tissues. (Figure 6C).

\section{miR-761 promoted the sensitivity of colorectal cancer cells to 5-FU through targeting FOXM1}

The expression level of FOXM1 was upregulated in the olorectal cancer cell (HT29, SW480, SW620 and DLD-1) compared to normal colon epithelium cell line (FHC) (Figure 7A). The expression of FOXM1 was upregulated in the HT29 cell after tranfected with FOXM1 vector (Figure 7B). The response of HT29 cell to 5-FU was enhanced after treated with FOXM1 vector compared with the cotrol-transfected cells (Figure 7C). Moreover, the response of miR-761-overexpressing HT29 cell to 5 -FU was rescued after transfection with the FOXM1 construct compared to the control vector (Figure 7D).

\section{miR-761 suppressed colorectal cancer cell proliferation and invasion by downregulating FOXM1}

Overexpression of FOXM1 rescued miR-761overexpressing HT29 cell proliferation (Figure 8A). In addition, elvelated expression of FOXM1 enhanced cyclin D1 expression in the miR-761-overexpressing HT29 cell (Figure 8B). Elevated expression of FOXM1 promoted the miR-761-overexpressing HT29 cell cycle (Figure 8C). Furthermore, miR-761 overexpression increased the miR761-overexpressing HT29 cell invasion (Figure 8D and 8E).

\section{DISCUSSION}

Chemoresistance is a great challenge for treating patients with advanced colorectal cancer $[30,31]$. It is important for us to find new strategies for conquering the resistance to chemotherapeutic events. In this study, we indicated that overexpression of miR-761 promoted 

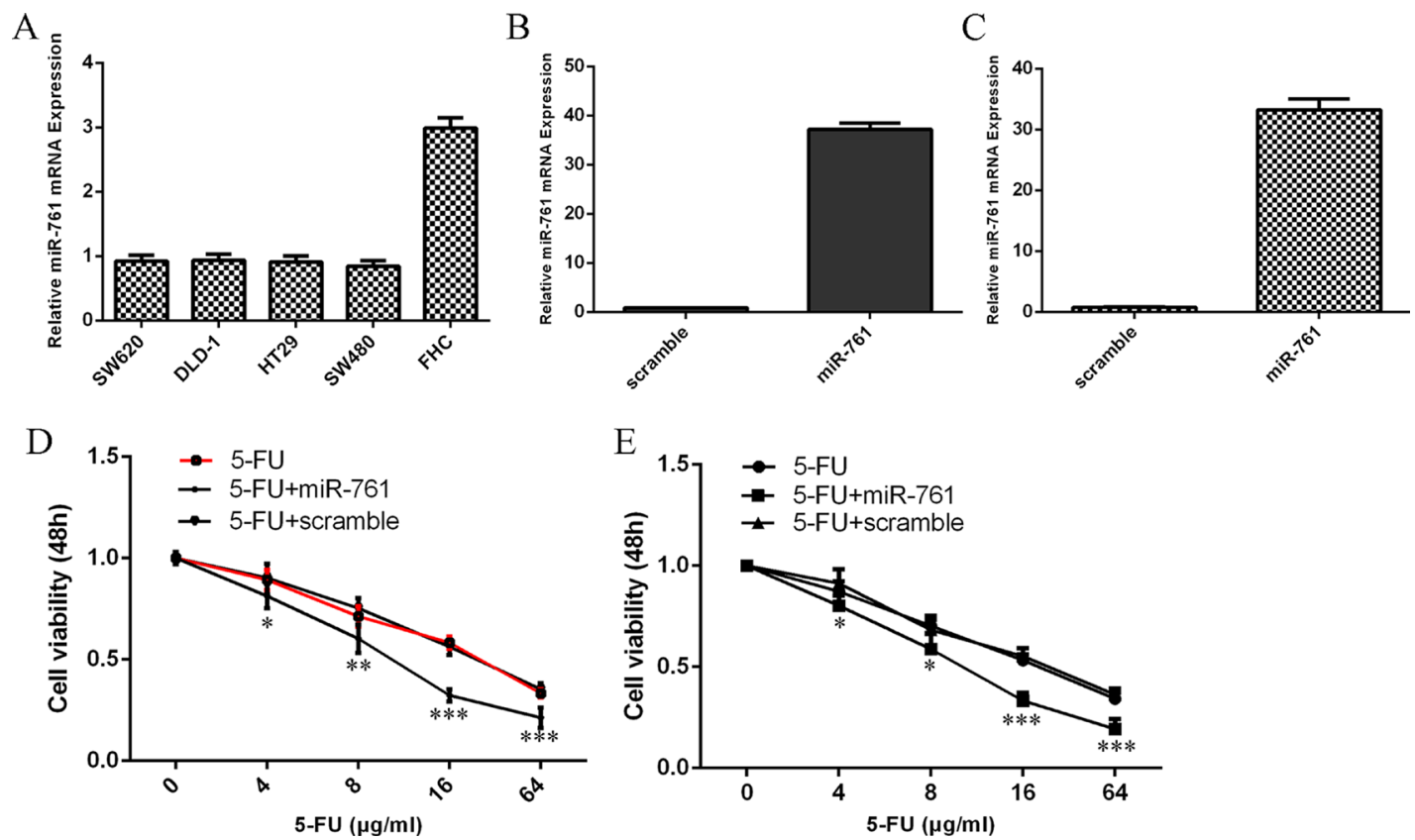

Figure 1: miR-761 promoted the sensitivity of colorectal cancer cells to 5-FU. (A) The miR-761 expression in the colorectal cancer cell (HT29, SW480, SW620 and DLD-1) and normal colon epithelium cell line (FHC) was detected by qRT-PCR analysis. U6 was used as the internal control. (B) The expression level of miR-761 in the HT29 cell treated with miR-761 mimic was measured by qRT-PCR analysis. (C) The expression level of SW480 in the HT29 cell treated with miR-761 mimic was measured by qRT-PCR analysis. (D) The response of HT29 cells to 5-FU was increased after treated with the miR-761 mimic compared to the scramble-transfected cells. (E) The response of SW480 cells to 5-FU was increased after treated with the miR-761 mimic compared to the scramble-transfected cells. ${ }^{*} p<0.05$; ${ }^{* *} p<0.01$ and ${ }^{* * *} p<0.001$.
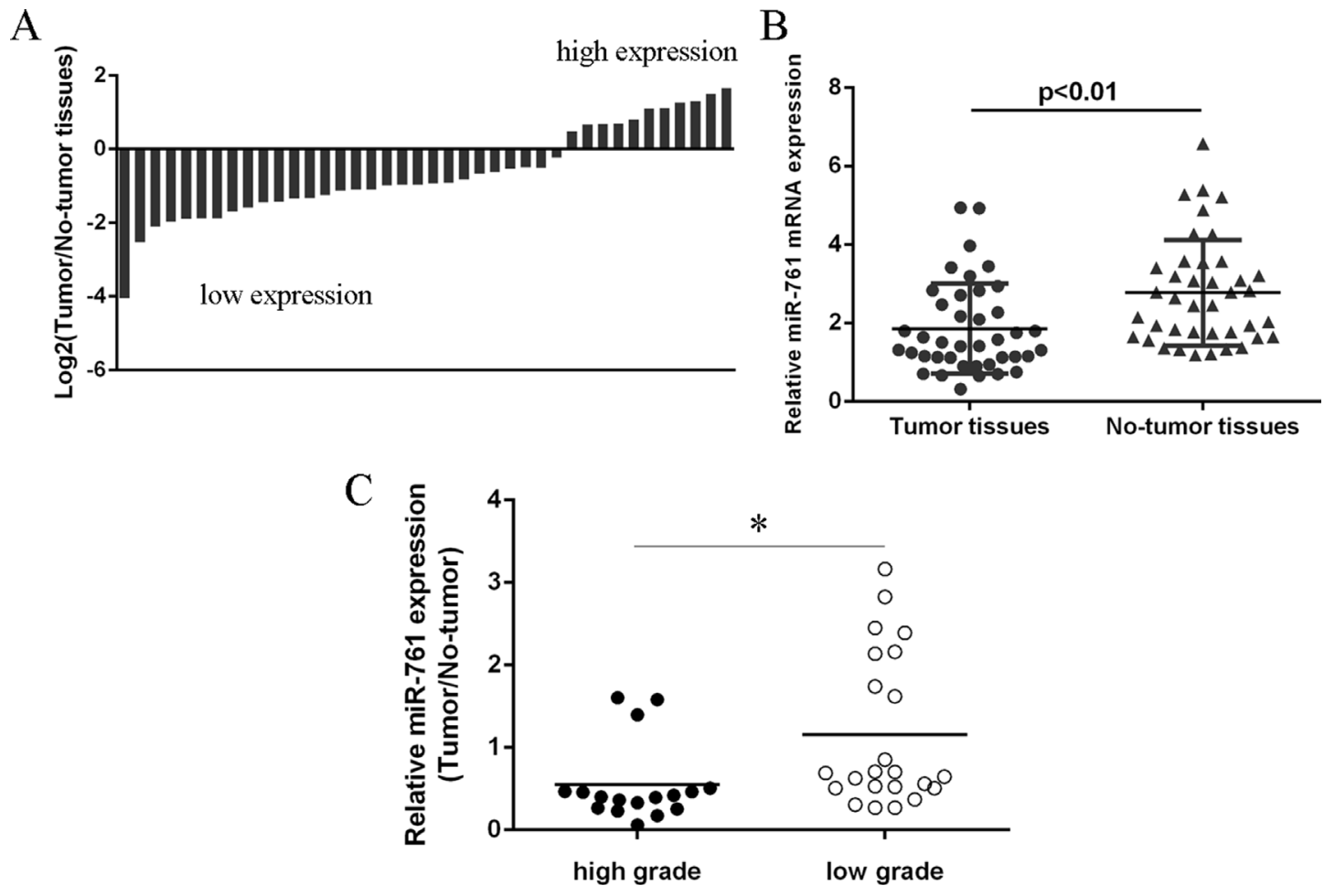

Figure 2: miR-761 expression was downregulated in colorectal cancer tissues. (A) The miR-761 expression in the colorectal cancer tissues and the adjacent non-tumor tissues was determined by qRT-PCR. U6 was used as the internal control. (B) The expression of miR-761 was lower in the colorectal cancer samples compare to the non-tumor samples. (C) The miR-761 expression was lower in the colorectal cancer patients with low grade than in the patients with low grade. ${ }^{*} p<0.05$. 
the sensitivity of colorectal cancer cells to 5-FU. We demonstrated that the expression level of miR-761 was downregulated in colorectal cancer cell (HT29, SW480, SW620 and DLD-1) compared to normal colon epithelium cell line (FHC). Furthermore, we showed that the expression of miR-761 was downregulated in colorectal cancer tissues. miR-761 expression was lower in the patients with low grade than in patients with high grade. In additon, we indicated that elevated expression of miR-761 suppressed colorectal cancer cell proliferation, cell cycle, colony formation and cell invasion. We identified that FOXM1 was a direct target gene of miR-761 in colorectal cancer cell. FOXM1 expression was upregulated in colorectal cancer tissues compare to the adjacent non-tumor tissues. miR-761 expression was negatively associated with the expression of FOXM1 in colorectal cancer tissues. Elevated expression of FOXM1 suppressed the sensitivity of miR761-overexpressing HT29 cells to 5-FU. We also indicated that FOXM1 overexpression promote cell proliferation, cycle and invasion of miR-761-overexpressing HT29 cells. These data suggested that miR-761 played a tumor suppressor miRNA in colorectal cancer progression and
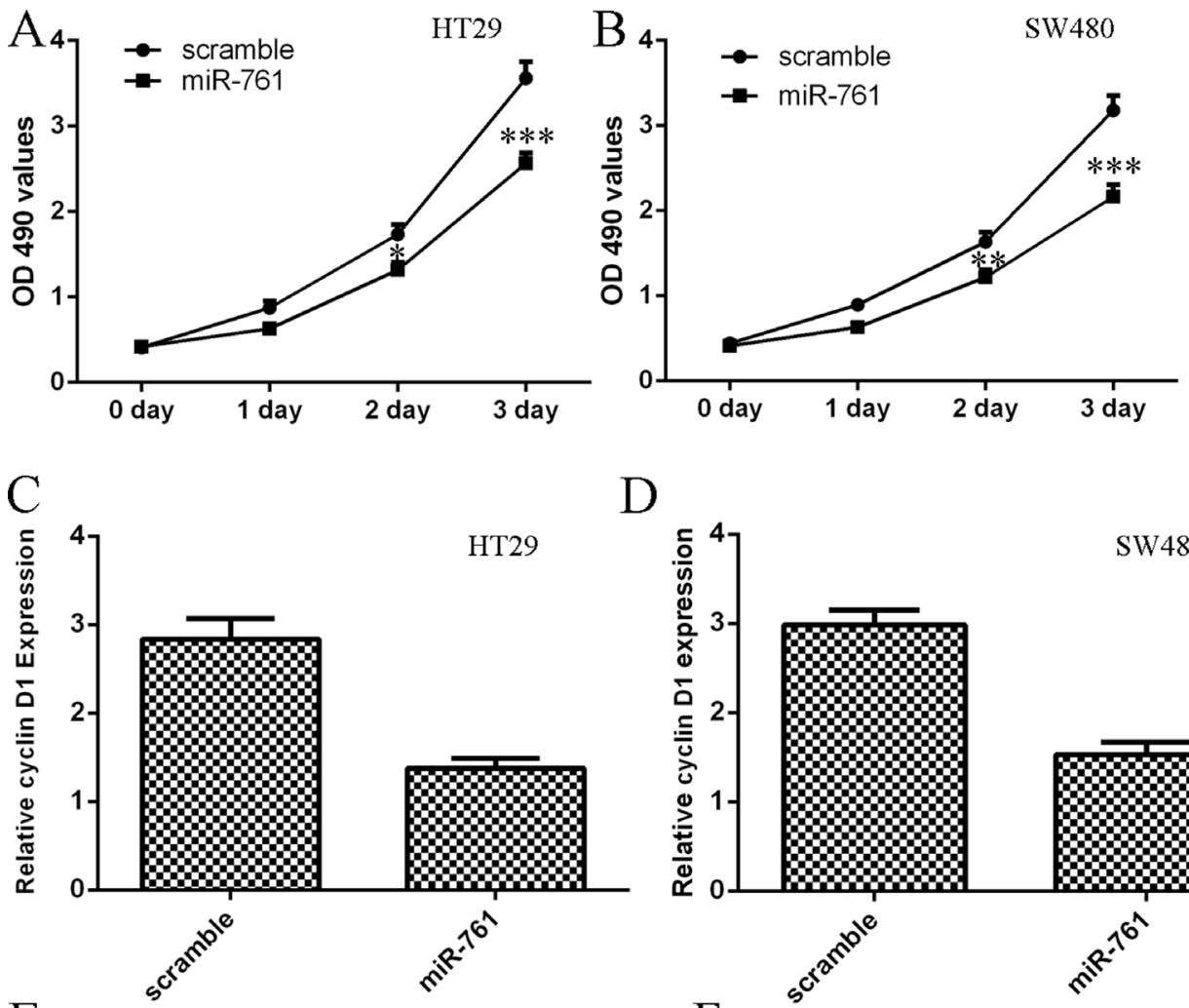

$\mathrm{D}$

$\mathrm{E}$

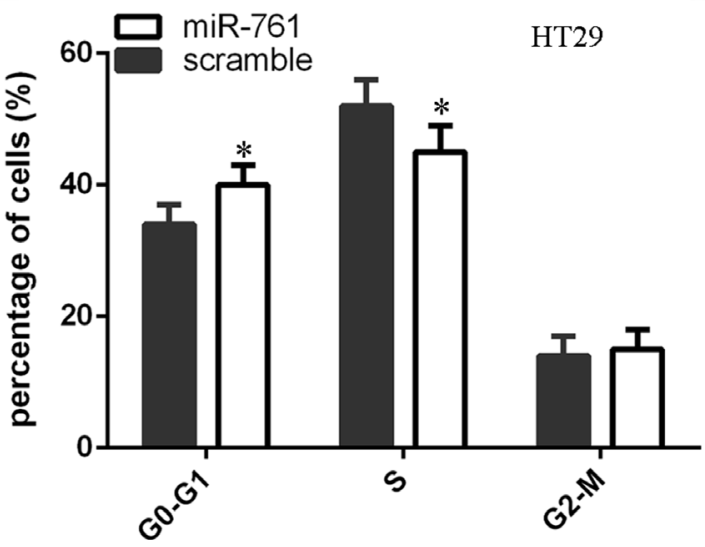

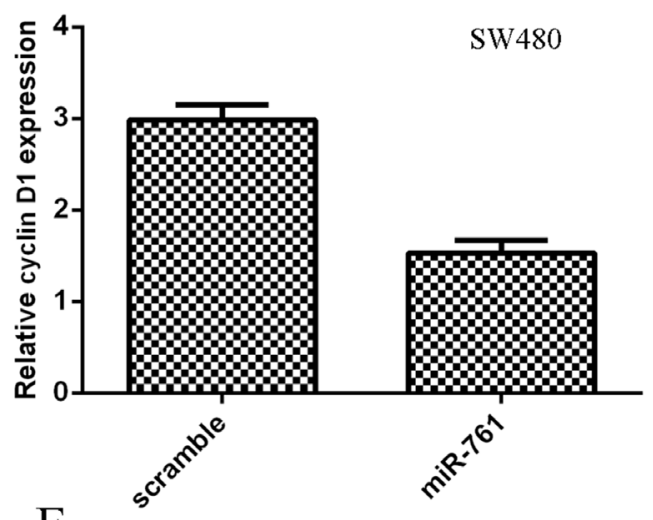

$\mathrm{F}$

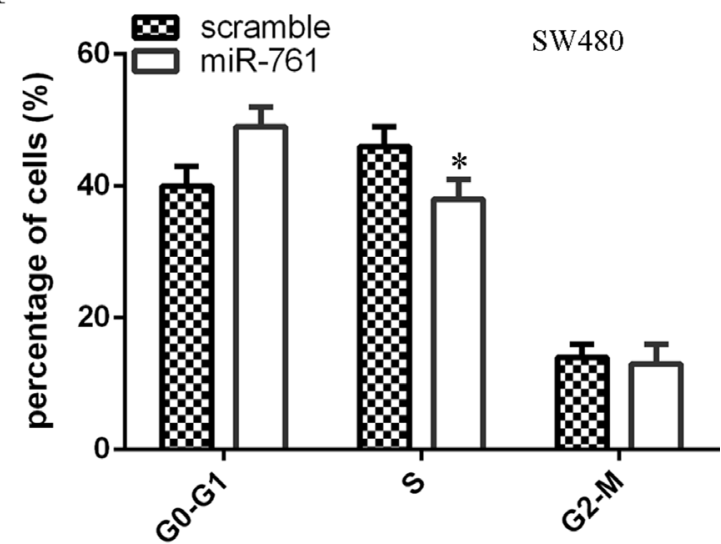

Figure 3: Elevated expression of miR-761 suppressed colorectal cancer cell proliferation. (A) Overexpression of miR-761 suppressed the HT29 cell proliferation. (B) Ectopic expression of miR-761 suppressed the SW480 cell proliferation. (C) Elevated expression of miR-761 decreased the cyclin D1 expression in the HT29 cell. (D) Overexpression of miR-761 decreased the cyclin D1 expression in the SW480 cell. (E) Ectopic expression of miR-761 decreased the HT29 cell cycle. (F) Elevated expression of miR-761 suppressed the SW480 cell cycle. ${ }^{*} p<0.05 ;{ }^{* *} p<0.01$ and ${ }^{* * *} p<0.001$. 
reduced miR-761 expression may be a major mechanism for 5-FU resistance in colorectal cancer cell.

Previous studies suggested that miR-761 acted important roles in the development and progression of various tumors [34-37]. Yan et al [34]. indicated that miR-761 expression was upregulated in nonsmall cell lung cancer (NSCLC) serum and tissues and overexpression of miR-761 increased NSCLC cell
A HT29 scramble

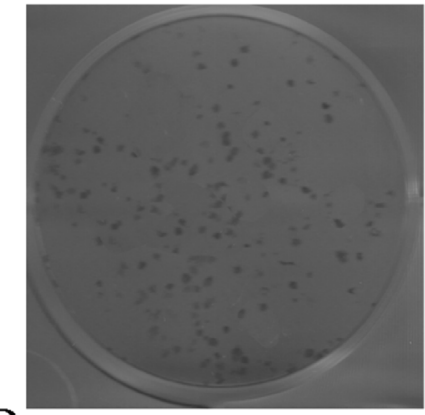

$\mathrm{B}_{\mathrm{SW} 480}$

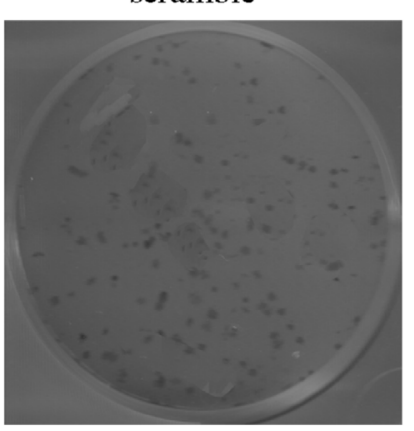

$\mathrm{C}$

HT29

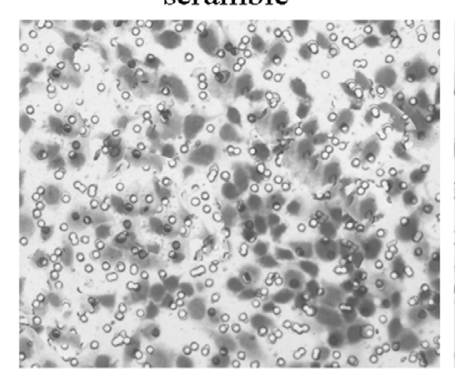

$\mathrm{D}$

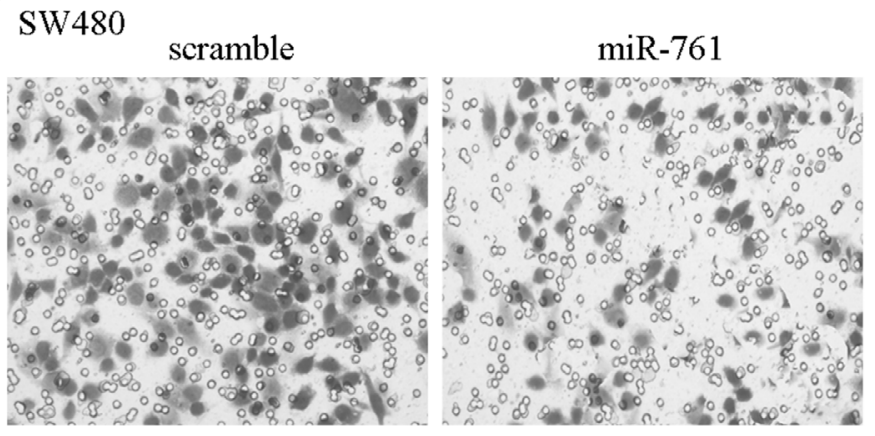

$\operatorname{miR}-761$

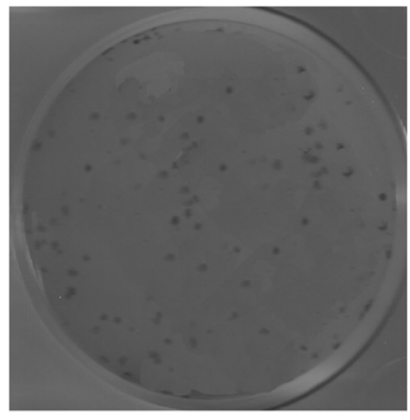

$\operatorname{miR}-761$

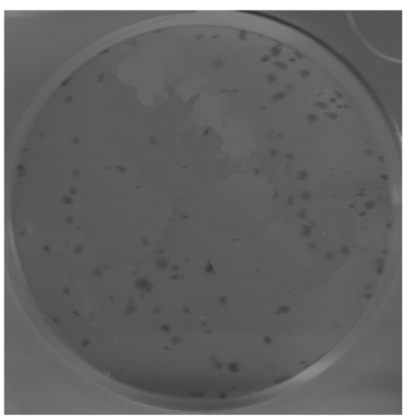

$\operatorname{miR}-761$

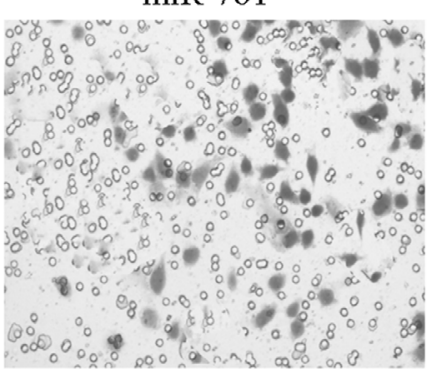

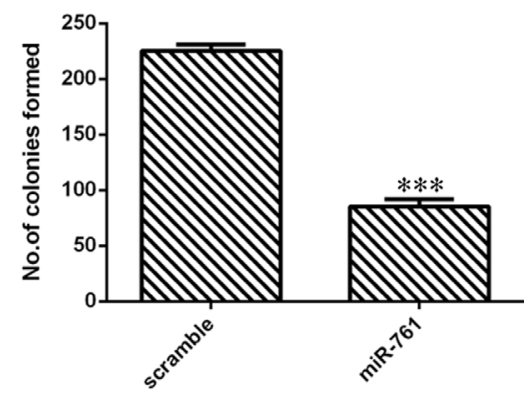
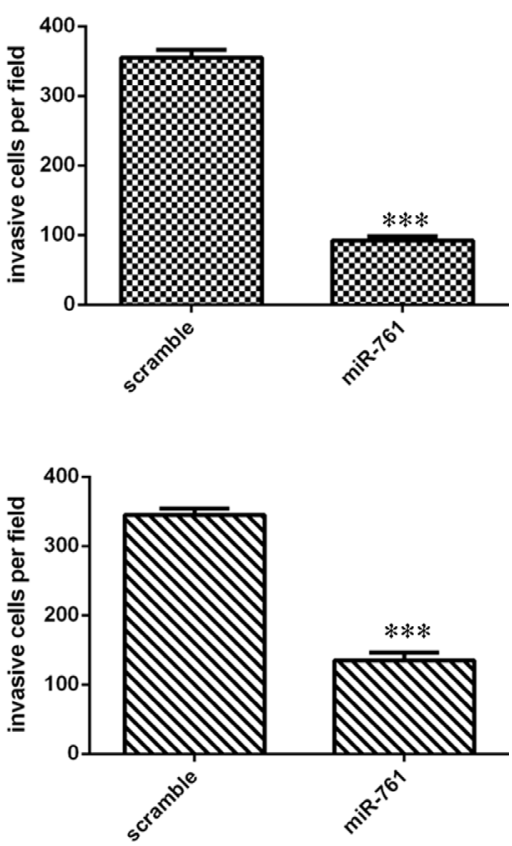

Figure 4: Overexpression of miR-761 decreased the colorectal cancer cell colony formation and invasion. (A) Ectopic expression of miR-761 suppressed the HT29 cell colony formation. The relative colony formation numbers were shown in the right. (B) Overexpression of miR-761 suppressed the SW480 cell colony formation. The relative colony formation numbers were shown in the right. (C) miR-761 overexpression inhibited the cell invasion in the HT29 cell. The relative invasive cells were shown. (D) Elevated expression of miR-761 suppressed the SW480 cell invasion. The relative invasive cells were shown. ${ }^{* * *} p<0.001$. 


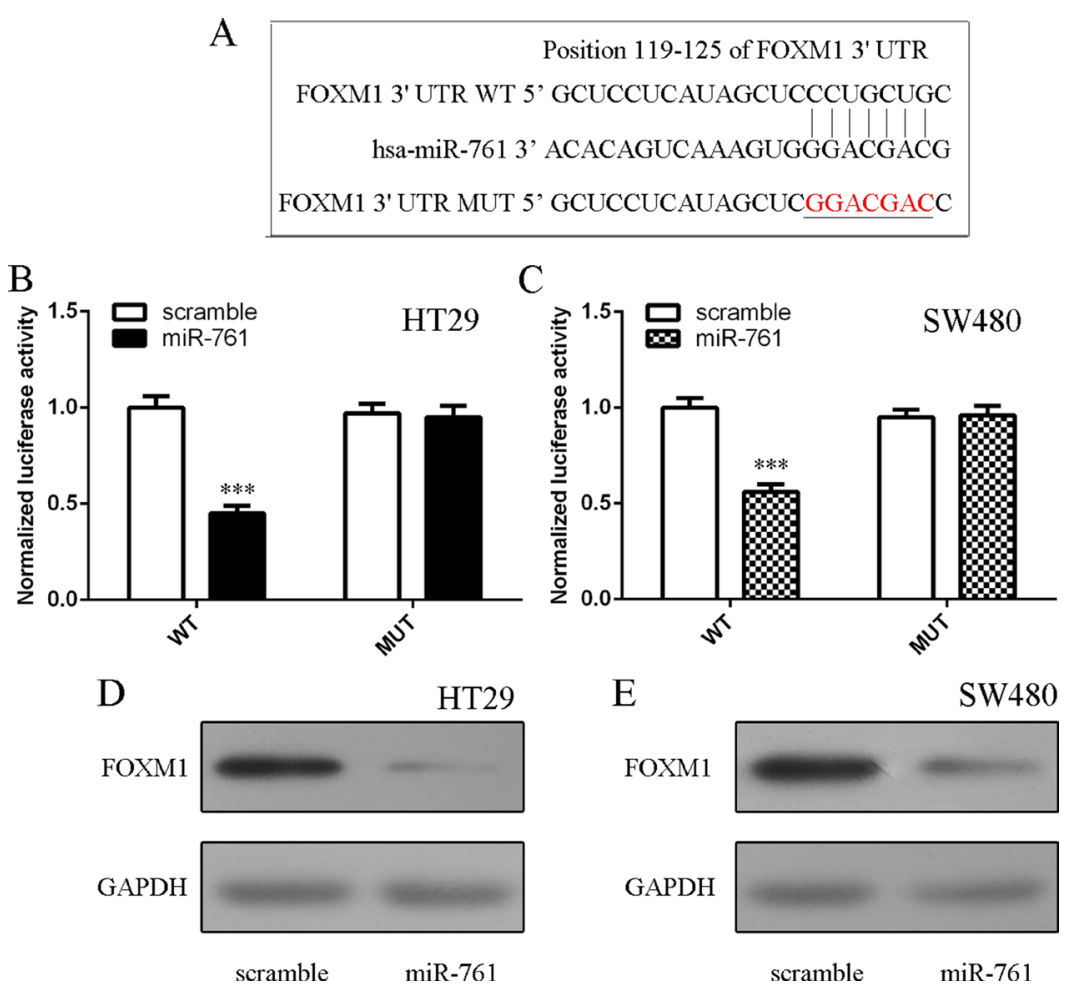

Figure 5: FOXM1 was a direct target gene of miR-761. (A) The putative binding sites of miR-761 and FOXM1 are shown. (B) Ectopic expression of miR-761 decreased the luciferase activity of wild-type 3'UTR of the FOXM1 construct, but not in the mutated-type 3'UTR of the FOXM1 vector in the HT29 cell. (C) Elevated expression of miR-761 decreased the luciferase activity of wild-type 3'UTR of the FOXM1 construct, but not in the mutated-type 3'UTR of the FOXM1 vector in the SW480 cell. (D) The protein expression of FOXM1 was measured by Western blot. GAPDH was used the internal control. (E) Elevated expression of miR-761 inhibited the protein expression of FOXM1 both in the SW480 cell. ${ }^{* * *} p<0.001$.
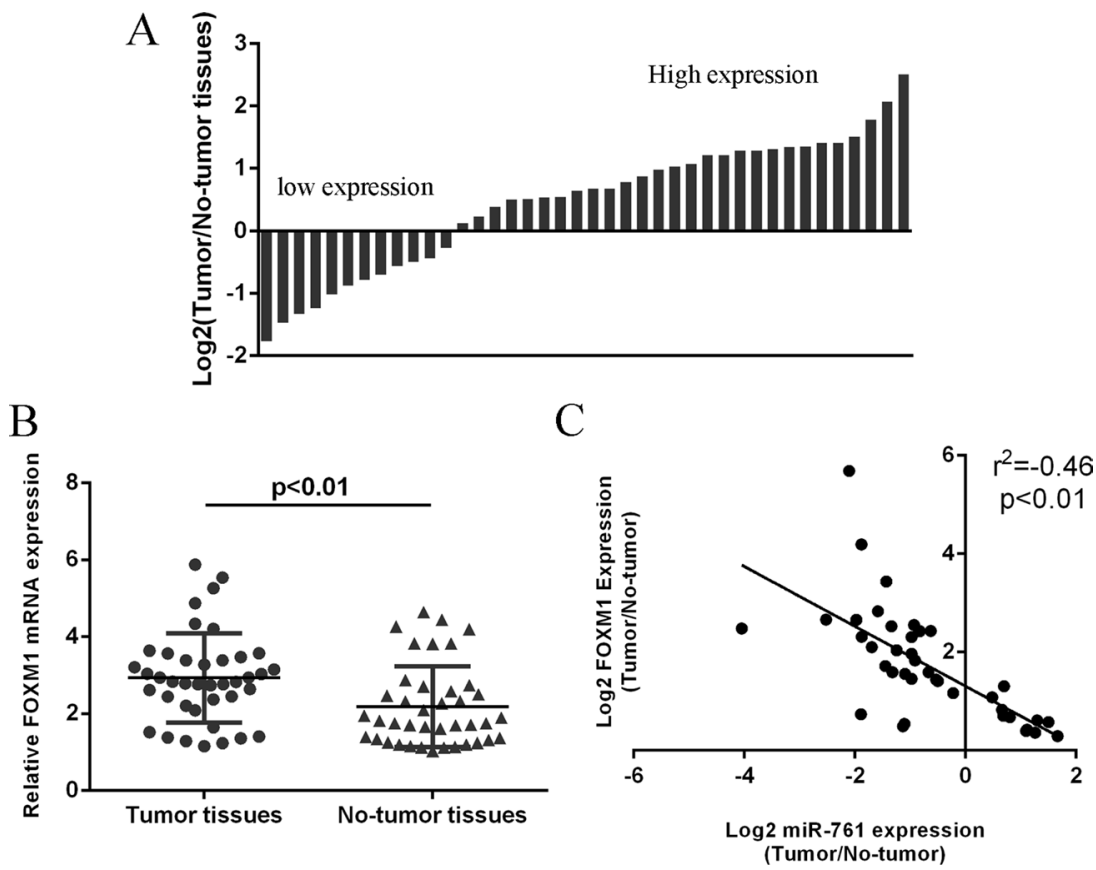

Figure 6: FOXM1 expression was upregulated in colorectal cancer tissues. (A) The FOXM1 expression in the colorectal cancer tissues and adjacent non-tumor tissues was measured by qRT-PCR and U6 was used as the internal control. (B) The expression of FOXM1 was higher in the colorectal cancer samples than in the non-tumor samples. (C) The miR-761 expression was negatively associated with the expression of FOXM1 in the colorectal cancer tissues. 

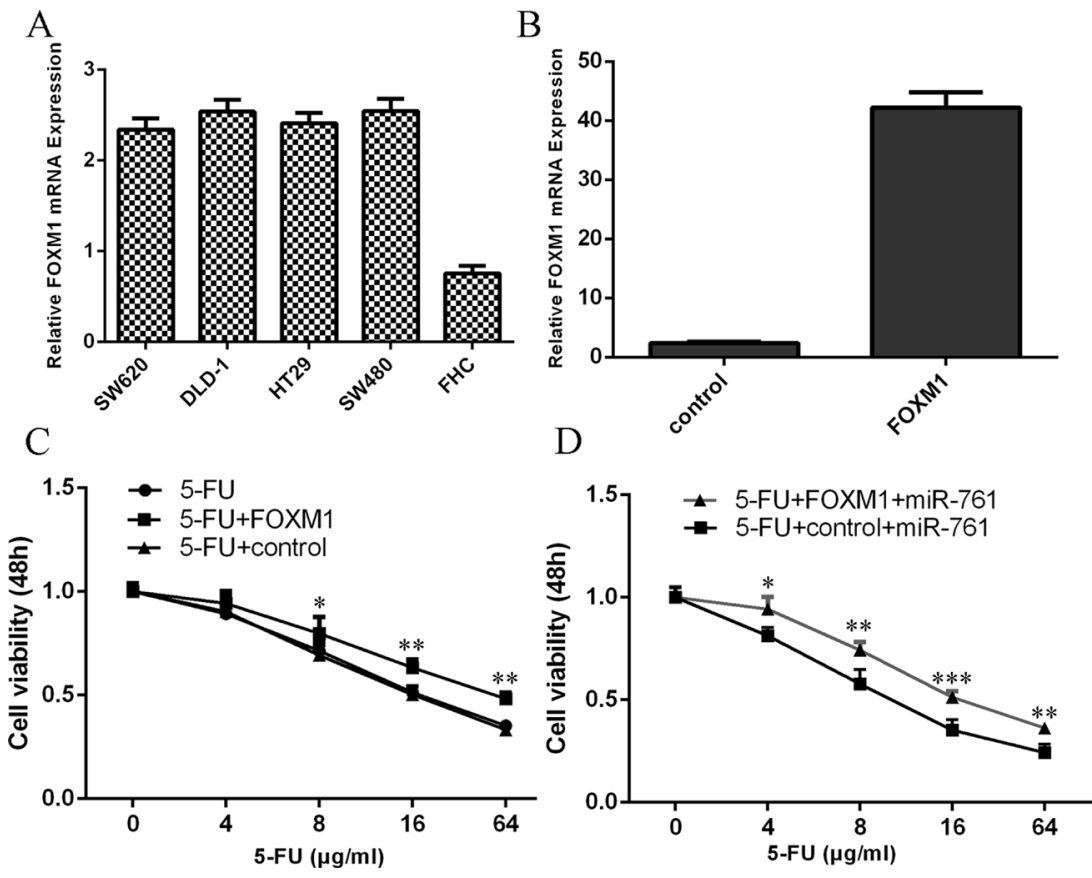

Figure 7: miR-761 promoted the sensitivity of colorectal cancer cells to 5-FU through targeting FOXM1. (A) The expression level of FOXM1 in the olorectal cancer cell (HT29, SW480, SW620 and DLD-1) and normal colon epithelium cell line (FHC) was measured by qRT-PCR. (B) The expression of FOXM1 in the HT29 cell with FOXM1 vector was determined by qRT-PCR. (C) The response of HT29 cell to 5-FU was enhanced after treated with the FOXM1 vector compared with the cotrol-transfected cells. (D) The response of miR-761-overexpressing HT29 cell to 5-FU was rescued after transfection with the FOXM1 construct compared to the control vector. ${ }^{*} p<0.05 ;{ }^{* *} p<0.01$ and ${ }^{* * *} p<0.001$.
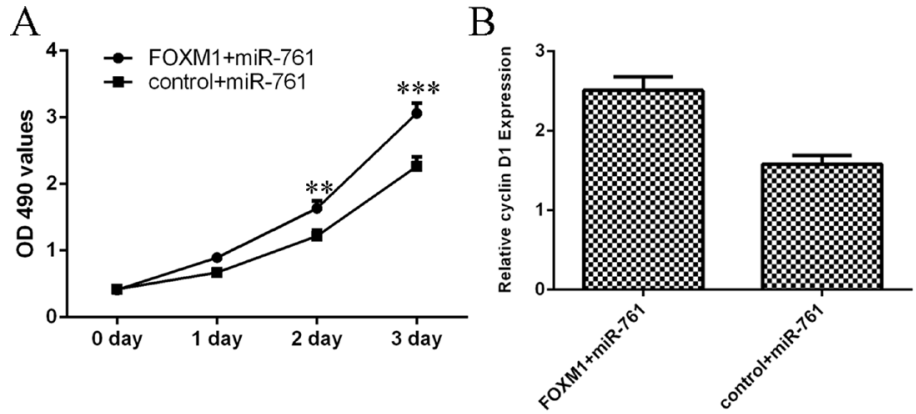

$\mathrm{D}$

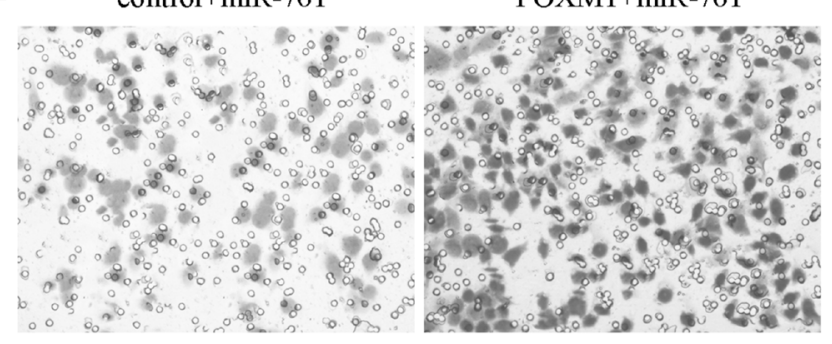

$\mathrm{C}$

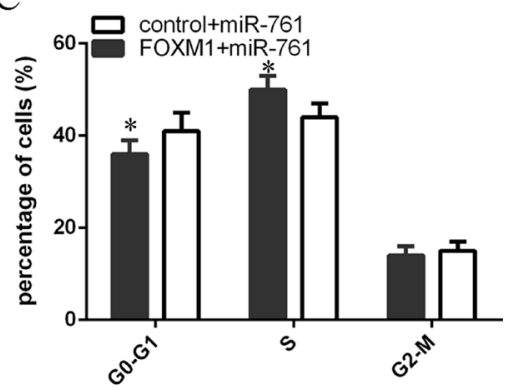

$\mathrm{E}$

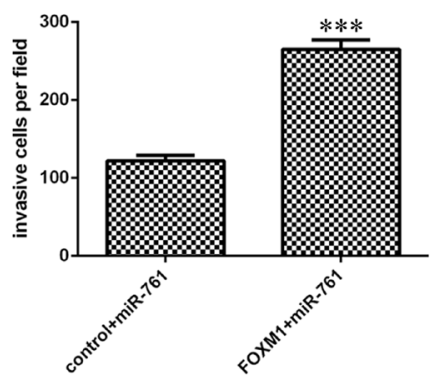

Figure 8: miR-761 suppressed the colorectal cancer cell proliferation and invasion by downregulating FOXM1. (A) Overexpression of FOXM1 rescued miR-761-overexpressing HT29 cell proliferation. (B) The mRNA expression of FOXM1 was measured by qRT-PCR. GAPDH was used as the internal control. (C) Elevated expression of FOXM1 promoted the miR-761-overexpressing HT29 cell cycle. (D) miR-761 overexpression increased the miR-761-overexpressing HT29 cell invasion. (E) The relative invasive cells were shown. ${ }^{*} p<0.05 ;{ }^{* *} p<0.01$ and ${ }^{* * *} p<0.001$. 
proliferation and metastasis through regulating ING4 and TIMP2 expression. Zhou et al [35]. found that miR-761 expression was upregulated in hepatocellular carcinoma tissues and inhibted expression of miR-761 impaired mitochondrial function and inhibited tumor metastasis and growth through upregulating Mitofusin-2. Guo et al [37]. demonstrated that miR-761 expression was increased in breast tumor tissues and cell lines and overexpression of miR-761 promoted cell proliferation, colony formation, invasion and migration through targeting tripartite motif-containing 29 (TRIM29). However, Shi et al [36]. demonstrated that the expression of miR-761 was downregulated in ovarian cancer tissues and miR-761 overexpression decreased ovarian cancer cell invasion and proliferation through inhibiting MSI1 expression. Until now, there are no references reported about the role of miR-761 in the development of colorectal cancer. In our study, we indicated that miR-761 expression was downregulated in colorectal cancer tissues. miR-761 expression was lower in patients with low grade than in patients with high grade CRC. Moreover, we indicated that elevated expression of miR-761 suppressed colorectal cancer cell proliferation, cell cycle, colony formation and cell invasion. Theses data suggested that miR-761 played as a tumor suppressor miRNA in colorectal cancer progression.

Forkhead box M1 (FOXM1) is a key transcription factor characterized by a winged-helix DNA-binding domain, which is also known as HNF-3/fork-head homolog 11, forkheaddrosophila homolog like 16 and Trident and membrane palmitoylated protein 2 [38-40]. Increasing studies showed that FOXM1 played critical roles in cell cycle, DNA replication, invasion, migration, angiogenesis and drug resistance [41-44]. FOXM1 expression was found to be upregulated in several tumors such as gastric cancer, hepatocellular carcinoma, lung caner, glioma and also colorectal cancer [42, 45-48]. For example, Zheng et al [49]. demonstrated that overexpression of FOXM1 promoted colorectal cancer cell invasion and migration through regulating pituitary tumor transforming gene (PTTG1) expression. Zhang et al [50]. also found that FOXM1 expression was upregulated in colorectal cancer tissues and silencing of FOXM1 decreased colorectal cancer cell migration and invasion. Recently, Xie et al. [41]. showed that the expression of FOXM1 was upregulated in the 5-FU nonresponsive CRC patients and inhibition of FOXM1 resensitized resistant $\mathrm{CRC}$ cells to 5-FU treatment through regulating ABCC10 expression. In addition, Liu et al. [51]. showed that miR149 promoted the sensitivity of colorectal cancer cell to 5-FU through targeting FOXM1. In this study, we also found that the expression of FOXM1 was upregulated in colorectal cancer cell lines and tissues. Overexpression of miR-761 suppressed luciferase activity of wild-type 3'UTR of the FOXM1 construct, but not in the mutatedtype 3'UTR of the FOXM1 vector. Ectopic expression of
miR-761 suppressed the FOXM1 expression. Furthermore, the miR-761 expression was negatively associated with the expression of FOXM1 in colorectal cancer tissues. In addition, miR-761 overexpression promoted the sensitivity of colorectal cancer cells to 5-FU through targeting FOXM1. Ectopic expression of miR-761 inhibited colorectal cancer cell proliferation and invasion by downregulating FOXM1 expression.

In summary, our data suggested that miR-761 was downregualted in colorectal cancer tissues and cells. Overexpression of miR-761 promoted the sensitivity of colorectal cancer cells to 5-FU and ectopic expression of miR-761 suppressed colorectal cancer cell proliferation, cell cycle, colony formation and invasion partly through regulating FOXM1 expression.

\section{MATERIALS AND METHODS}

\section{Tissue samples and cell culture and transfection}

The colorectal tumor tissues and adjacent normal samples utilized in our study were collected from our department. Informed consents were obtained from each patients and this experiment was approved by the review board of The Second Affiliated Hospital and Yuying Children's Hospital of Wenzhou Medical University. Four colorectal tumor cell lines (HT29, SW480, SW620 and DLD-1) and one normal colon epithelium cell line (FHC) were purchased from the ATCC (MD, USA) and were keplt in the Dulbecco's modified Eagle's medium (DMEM). miR-761 and scramble, pcDNA-FOXM1 and control vector were synthesized from the GenePharma Company (Shanghai, China). Colorectal tumor cells were transfected with plasmid by using the Lipofectamin 2000 (Invitrogen, CA, USA) following to the manufacturer's information.

\section{RNA extraction and qRT-PCR}

Total RNA was extracted from cell or sample using the Trizol reagent (Invitrogen, CA, USA). Quantitative RTPCR (qRT-PCR) was conducted in the Bio-Rad (Hercules, USA) IQ5 PCR system following to the manufacturer's instruction to measure the miR-761 and FOXM1 expression. The qPCR reaction was done with the following: 15 minutes at $50^{\circ} \mathrm{C}, 10$ minutes at $95^{\circ} \mathrm{C}$, followed by 42 cycles at the $95^{\circ} \mathrm{C}$ for 15 second and $60^{\circ} \mathrm{C}$ for 1 minute. U6 and GAPDH were conducted as the internal control for miR761 and FOXM1 respectively. The sequences are listed as follows: miR-761 forward 5'-ACAGCAGGCACAGAC-3' and reverse 5'-GAGCAGGCTGGAGAA-3'; FOXM1 forward 5'-GTGAATGGTCCAGAAGGAGAC-3' and reverse 5'-ACCACTTTCCCTACTTTAAGCAC-3'; U6 forward 5'-CTCGCTTCGGCAGCACATATACT-3' and reverse 5'- ACGCTTCACGAATTTGCGTGTC-3'; and GAPDH were forward 5'- TGTTCGACAGTCAGCCGC-3' and reverse 5'-GGTGTCTGAGCGATGTGGC-3'. 


\section{Western blot}

Western blot assay was done to measure the protein expression of FOXM1. The protein lysate was extracted from cells or samples using the Protein Extraction Reagent (Thermo Fisher Scientific, USA). The protei lysate was separated by $10 \%$ SDS-PAGE gel and transfermed to PVDF membrane (Millipore). The membranes were blocked with $5 \%$ non-fat milk and then incubated with the fisrt antibodies (FOXM1 and GAPDH, 1:1000 dilution, Abcam). GAPDH was performed as the internal control for candidate genes.

\section{Luciferase reporter assay}

The HT29 and SW480 cell were co-transfected with the pGL-3 FOXM1 reporter construct, pGL-3 control vector and miR-761 or the scramble vector using the Lipofectamin 2000 (Invitrogen, CA, USA) according to the manufacturer's information. The cell was harvested 24 hours post-transfection and the luciferase reporter assay was measured by the Dual Luciferase Assay (Promega, WI, USA) following to the manufacturer's information.

\section{Cell proliferation, invasion and colony formation assays}

Cell proliferation was determined using the $3^{\prime}(4$, 5-dimethylthazol-2-yl)-2, 5-diphenyltetrazolium bromide (MTT) analysis. The cells were cultured into 96 well plates and cell viability was measured the MTT assay according to manufacturer's information. The absorbance at the 490 $\mathrm{nm}$ was read on the spectrophotometer. For cell invasion assay, cell was placed on the upper chamber with Matrigel coated membrane which was added to the serum-free culture medium. In the lower chamber, 10\% FBS (fetal bovine serum) was added. After incubation for 24 hours, cell that invased to the bottom of the chamber was fixed with methanol and stained with crystal violet. For cell colony formation assay, the cell was cultured on the 6-well plate and continuted to culture for 2 weeks. The colony cell was then stained with the crystal violet and counted.

\section{Statistical analysis}

The statistical analysis in our study was measured using the SPSS 17.0 software. The numerical data was shown as mean $\pm \mathrm{SD}$ (standard deviation). The difference between more than two groups was performed with the one-way ANOVA. Student's $t$-test was used to assess the difference between two groups. $P<0.05$ was considered as significant.

\section{CONFLICTS OF INTEREST}

None.

\section{REFERENCES}

1. Wang L, Qian L, Li X, Yan J. MicroRNA-195 inhibits colorectal cancer cell proliferation, colony-formation and invasion through targeting CARMA3. Molecular medicine reports. 2014; 10:473-478.

2. Balaguer F, Link A, Lozano JJ, Cuatrecasas M, Nagasaka T, Boland CR, Goel A. Epigenetic silencing of miR-137 is an early event in colorectal carcinogenesis. Cancer research. 2010; 70:6609-6618.

3. Burum-Auensen E, DeAngelis PM, Schjolberg AR, Roislien J, Mjaland O, Clausen OP. Reduced level of the spindle checkpoint protein BUB1B is associated with aneuploidy in colorectal cancers. Cell proliferation. 2008; 41:645-659.

4. Mansour MA, Hyodo T, Ito S, Kurita K, Kokuryo T, Uehara K, Nagino M, Takahashi M, Hamaguchi M, Senga T. SATB2 suppresses the progression of colorectal cancer cells via inactivation of MEK5/ERK 5 signaling. The FEBS journal. 2015; 282:1394-1405.

5. Perilli L, Vicentini C, Agostini M, Pizzini S, Pizzi M, D’Angelo E, Bortoluzzi S, Mandruzzato S, Mammano E, Rugge M, Nitti D, Scarpa A, Fassan M, et al. Circulating miR-182 is a biomarker of colorectal adenocarcinoma progression. Oncotarget. 2014; 5:6611-6619. https://doi. org/10.18632/oncotarget.2245.

6. Liu GH, Zhou ZG, Chen R, Wang MJ, Zhou B, Li Y, Sun XF. Serum miR-21 and miR-92a as biomarkers in the diagnosis and prognosis of colorectal cancer. Tumour biology. 2013; 34:2175-2181.

7. Yamashita S, Yamamoto H, Mimori K, Nishida N, Takahashi H, Haraguchi N, Tanaka F, Shibata K, Sekimoto M, Ishii H, Doki Y, Mori M. MicroRNA-372 is associated with poor prognosis in colorectal cancer. Oncology. 2012; 82:205-212.

8. Xu XM, Qian JC, Deng ZL, Cai Z, Tang T, Wang P, Zhang KH, Cai JP. Expression of miR-21, miR-31, miR-96 and miR-135b is correlated with the clinical parameters of colorectal cancer. Oncology letters. 2012; 4:339-345.

9. Wu W, Wang Z, Yang P, Yang J, Liang J, Chen Y, Wang H, Wei G, Ye S, Zhou Y. MicroRNA-135b regulates metastasis suppressor 1 expression and promotes migration and invasion in colorectal cancer. Molecular and cellular biochemistry. 2013.

10. Toiyama Y, Takahashi M, Hur K, Nagasaka T, Tanaka K, Inoue Y, Kusunoki M, Boland CR, Goel A. Serum miR-21 as a diagnostic and prognostic biomarker in colorectal cancer. Journal of the National Cancer Institute. 2013; 105:849-859.

11. Liu H, Du L, Wen Z, Yang Y, Li J, Wang L, Zhang X, Liu Y, Dong Z, Li W, Zheng G, Wang C. Up-regulation of miR-182 expression in colorectal cancer tissues and its prognostic value. International journal of colorectal disease. 2013; 28:697-703.

12. Liu M, Lang N, Qiu M, Xu F, Li Q, Tang Q, Chen J, Chen X, Zhang S, Liu Z, Zhou J, Zhu Y, Deng Y, et al. miR-137 
targets Cdc42 expression, induces cell cycle G1 arrest and inhibits invasion in colorectal cancer cells. International journal of cancer. 2011; 128:1269-1279.

13. Li Z, Yu X, Shen J, Jiang Y. MicroRNA dysregulation in uveal melanoma: a new player enters the game. Oncotarget. 2015; 6:4562-4568. https://doi.org/10.18632/ oncotarget.2923.

14. Li Z, Yu X, Shen J, Wu WK, Chan MT. MicroRNA expression and its clinical implications in Ewing's sarcoma. Cell proliferation. 2015; 48:1-6.

15. Li Z, Yu X, Shen J, Liu Y, Chan MT, Wu WK. MicroRNA dysregulation in rhabdomyosarcoma: a new player enters the game. Cell proliferation. 2015; 48:511-516.

16. Huang $\mathrm{K}$, Dong $\mathrm{X}$, Sui $\mathrm{C}, \mathrm{Hu} \mathrm{D}$, Xiong $\mathrm{T}$, Liao $\mathrm{S}$, Zhang H. MiR-223 suppresses endometrial carcinoma cells proliferation by targeting IGF-1R. American journal of translational research. 2014; 6:841-849.

17. Yang Z, Han Y, Cheng K, Zhang G, Wang X. miR-99a directly targets the mTOR signalling pathway in breast cancer side population cells. Cell proliferation. 2014; 47:587-595.

18. Yin Z, Ding H, He E, Chen J, Li M. Up-regulation of microRNA-491-5p suppresses cell proliferation and promotes apoptosis by targeting FOXP4 in human osteosarcoma. Cell proliferation. 2016.

19. Yu Z, Zhang Y, Gao N, Wang X. Overexpression of miR-506 inhibits growth of osteosarcoma through Snail2. American journal of translational research. 2015; 7:2716-2723.

20. Chen HY, Lin YM, Chung HC, Lang YD, Lin CJ, Huang J, Wang WC, Lin FM, Chen Z, Huang HD, Shyy JY, Liang JT, Chen RH. miR-103/107 promote metastasis of colorectal cancer by targeting the metastasis suppressors DAPK and KLF4. Cancer research. 2012; 72:3631-3641.

21. Chen DL, Wang DS, Wu WJ, Zeng ZL, Luo HY, Qiu MZ, Ren C, Zhang DS, Wang ZQ, Wang FH, Li YH, Kang TB, $\mathrm{Xu} \mathrm{RH}$. Overexpression of paxillin induced by miR-137 suppression promotes tumor progression and metastasis in colorectal cancer. Carcinogenesis. 2013; 34:803-811.

22. Tang JT, Wang JL, Du W, Hong J, Zhao SL, Wang YC, Xiong H, Chen HM, Fang JY. MicroRNA 345, a methylation-sensitive microRNA is involved in cell proliferation and invasion in human colorectal cancer. Carcinogenesis. 2011; 32:1207-1215.

23. Gao Y, Xue Q, Wang D, Du M, Zhang Y, Gao S. miR-873 induces lung adenocarcinoma cell proliferation and migration by targeting SRCIN1. American journal of translational research. 2015; 7:2519-2526.

24. Shen L, Wang P, Yang J, Li X. MicroRNA-217 Regulates WASF3 Expression and Suppresses Tumor Growth and Metastasis in Osteosarcoma. PloS one. 2014; 9:e109138.

25. Zhang Y, Zheng D, Xiong Y, Xue C, Chen G, Yan B, Ye Q. miR-202 suppresses cell proliferation in human hepatocellular carcinoma by downregulating LRP6 posttranscriptionally. FEBS letters. 2014; 588:1913-1920.
26. Kang M, Ren MP, Zhao L, Li CP, Deng MM. miR-485-5p acts as a negative regulator in gastric cancer progression by targeting flotillin-1. American journal of translational research. 2015; 7:2212-2222.

27. Han Y, Liu Y, Zhang H, Wang T, Diao R, Jiang Z, Gui Y, Cai Z. Hsa-miR-125b suppresses bladder cancer development by down-regulating oncogene SIRT7 and oncogenic long noncoding RNA MALAT1. FEBS letters. 2013.

28. Jin K, Xiang Y, Tang J, Wu G, Li J, Xiao H, Li C, Chen Y, Zhao J. miR-34 is associated with poor prognosis of patients with gallbladder cancer through regulating telomere length in tumor stem cells. Tumour biology. 2014; 35:1503-1510.

29. Zhang Y, Zheng L, Huang J, Gao F, Lin X, He L, Li D, Li Z, Ding Y, Chen L. MiR-124 Radiosensitizes human colorectal cancer cells by targeting PRRX1. PloS one. 2014; 9:e93917.

30. Zhou Y, Wan G, Spizzo R, Ivan C, Mathur R, Hu X, Ye X, Lu J, Fan F, Xia L, Calin GA, Ellis LM, Lu X. miR-203 induces oxaliplatin resistance in colorectal cancer cells by negatively regulating ATM kinase. Molecular oncology. 2014; 8:83-92.

31. Nishida N, Yamashita S, Mimori K, Sudo T, Tanaka F, Shibata K, Yamamoto H, Ishii H, Doki Y, Mori M. MicroRNA-10b is a Prognostic Indicator in Colorectal Cancer and Confers Resistance to the Chemotherapeutic Agent 5-Fluorouracil in Colorectal Cancer Cells. Annals of surgical oncology. 2012; 19:3065-3071.

32. Ning FL, Wang F, Li ML, Yu ZS, Hao YZ, Chen SS. MicroRNA-182 modulates chemosensitivity of human non-small cell lung cancer to cisplatin by targeting PDCD4. Diagnostic pathology. 2014; 9:143.

33. Qin J, Luo M, Qian H, Chen W. Upregulated miR-182 increases drug resistance in cisplatin-treated HCC cell by regulating TP53INP1. Gene. 2014; 538:342-347.

34. Yan A, Yang CY, Chen ZB, Li CH, Cai L. MiR-761 Promotes Progression and Metastasis of Non-Small Cell Lung Cancer by Targeting ING4 and TIMP2. Cellular Physiology and Biochemistry. 2015; 37:55-66.

35. Zhou XH, Zhang LS, Zheng BC, Yan YC, Zhang Y, Xie HY, Zhou L, Zheng SS, Wang WL. MicroRNA-761 is upregulated in hepatocellular carcinoma and regulates tumorigenesis by targeting Mitofusin-2. Cancer science. 2016; 107:424-432.

36. Shi C, Zhang ZY. miR-761 inhibits tumor progression by targeting MSI1 in ovarian carcinoma. Tumor Biol. 2016; 37:5437-5443.

37. Guo GC, Wang JX, Han ML, Zhang LP, Li L. microRNA-761 induces aggressive phenotypes in triplenegative breast cancer cells by repressing TRIM29 expression. Cell Oncol. 2017; 40:157-166.

38. Liu Y, Wang Y, Gong X, Liang Y, Guo JF, Yao S. FoxM1 is involved in the development of acute myeloid leukemia by targeting Bcl-2 expression. International journal of clinical and experimental pathology. 2017; 10:4918-4926. 
39. Han J, Cui JF, Chen PX, Zhang H, Zhu YM, Zhang NS, Wang MM, Rasool K, Yu JM. Prognostic significance of FOXM1 in digestive system cancers: a systematic review and meta-analysis. International journal of clinical and experimental medicine. 2017; 10:4244-4255.

40. Molinuevo R, Freije A, de Pedro I, Stoll SW, Elder JT, Gandarillas A. FOXM1 allows human keratinocytes to bypass the oncogene-induced differentiation checkpoint in response to gain of MYC or loss of p53. Oncogene. 2017; 36:956-965.

41. Xie T, Geng J, Wang Y, Wang LY, Huang MX, Chen J, Zhang K, Xue LJ, Liu XB, Mao XB, Chen YA, Wang Q, Dai TT, et al. FOXM1 evokes 5-fluorouracil resistance in colorectal cancer depending on ABCC10. Oncotarget. 2017; 8:8574-8589. https://doi.org/10.18632/oncotarget.14351.

42. Shang RZ, Pu M, Li Y, Wang DS. FOXM1 regulates glycolysis in hepatocellular carcinoma by transactivating glucose transporter 1 expression. Oncology reports. 2017; 37:2261-2269.

43. Chan DW, Hui WWY, Wang JJ, Yung MMH, Hui LMN, Qin Y, Liang RR, Leung THY, Xu D, Chan KKL, Yao KM, Tsang BK, Ngan HYS. DLX1 acts as a crucial target of FOXM1 to promote ovarian cancer aggressiveness by enhancing TGF-beta/SMAD4 signaling. Oncogene. 2017; 36:1404-1416.

44. Song X, Kenston SSF, Zhao JS, Yang DT, Gu YL. Roles of FoxM1 in cell regulation and breast cancer targeting therapy. Medical Oncology. 2017; 34.

45. He Y, Yu YC, Zhu HC, Jing XJ, Wei N, Wang PD, Sun QS. MicroRNA-146 inhibits epithelial-mesenchymal transition by regulating FOXM1 in lung cancer cells. International journal of clinical and experimental pathology. 2017; 10:3085-3092.

46. Zhang C, Wang Y, Feng YF, Zhang Y, Ji B, Wang S, Sun Y, Zhu CY, Zhang DS, Sun YM. Gli1 promotes colorectal cancer metastasis in a Foxm1-dependent manner by activating EMT and PI3K-AKT signaling. Oncotarget. 2016; 7:86134-86147. https://doi.org/10.18632/oncotarget.13348.

47. Xu MD, Wang YQ, Weng WW, Wei P, Qi P, Zhang QY, Tan C, Ni SJ, Dong L, Yang YS, Lin WR, Xu QH, Huang D, et al. A Positive Feedback Loop of lncRNA-PVT1 and FOXM1 Facilitates Gastric Cancer Growth and Invasion. Clinical Cancer Research. 2017; 23:2071-2080.

48. Zhang S, Zhao BS, Zhou A, Lin K, Zheng S, Lu Z, Chen Y, Sulman EP, Xie K, Bögler O, Majumder S, He C, Huang S. m6A Demethylase ALKBH5 Maintains Tumorigenicity of Glioblastoma Stem-like Cells by Sustaining FOXM1 Expression and Cell Proliferation Program. Cancer Cell. 2017; 31:591-606.e6.

49. Zheng Y, Guo JJ, Zhou JJ, Lu JJ, Chen Q, Zhang C, Qing C, Koeffler HP, Tong YG. FoxM1 transactivates PTTG1 and promotes colorectal cancer cell migration and invasion. BMC medical genomics. 2015; 8:49.

50. Zhang HM, Zhong H, Li L, Ji WS, Zhang XQ. Overexpressed transcription factor FOXM1 contributes to the progression of colorectal cancer. Molecular medicine reports. 2016; 13:2696-2700.

51. Liu XB, Xie T, Mao XB, Xue LJ, Chu XY, Chen LB. MicroRNA-149 Increases the Sensitivity of Colorectal Cancer Cells to 5-Fluorouracil by Targeting Forkhead Box Transcription Factor FOXM1. Cellular Physiology and Biochemistry. 2016; 39:617-629. 